\title{
A case report of giant hamartoma of both kidneys with spontaneous rupture and hemorrhage in a pregnant woman: A case report
}

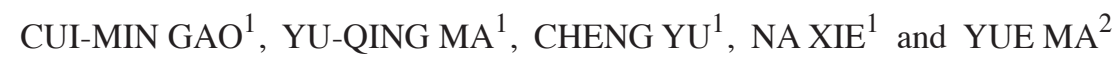 \\ ${ }^{1}$ Department of Anesthesiology, First Hospital of Lanzhou University; ${ }^{2}$ Department of Clinical Medicine, \\ First College of Clinical Medicine of Lanzhou University, Lanzhou, Gansu 730000, P.R. China
}

Received February 12, 2019; Accepted June 18, 2019

DOI: $10.3892 / b r .2019 .1223$

\begin{abstract}
The aim of the present study was to describe a case of renal angiomyolipoma (RAML) in a 31-year-old woman who presented with massive hemorrhage, shock, severe anemia (Hb $63 \mathrm{~g}-\mathrm{l})$ and multiple lesions, prior to delivery of a dead fetus. A 31-year-old woman was admitted due to left flank and abdominal pain at 20 weeks of gestation age, and diagnosed with RAML complicated with spontaneous rupture and hemorrhage of the left kidney, for which emergency exploratory laparotomy, left kidney resection and splenectomy were performed. The patient delivered a dead fetus 3 days following surgery and recovered well postoperatively. Hemorrhagic RAML during pregnancy is a rare and complex vascular surgical emergency, and should be managed in a multidisciplinary manner. Spontaneous rupture is a serious threat to the life and health of pregnant women and fetuses. The present case is a typical example of RAML in a pregnant woman complicated by spontaneous rupture and hemorrhage, which highlights the importance of determining the risk of acute hemorrhage in early stages of pregnancy, and the significance of time and proper management. However, in cases of shock caused by spontaneous rupture and hemorrhage, the only way to save the life of the patient is to resect the lesion without delay.
\end{abstract}

\section{Introduction}

Renal angiomyolipoma (RAML), also known as renal hamartoma, is a benign tumor composed of mature adipose tissue, smooth muscle and thick-walled blood vessels, which may impose a significant morbidity or mortality due to its unique

Correspondence to: Professor Yu-Qing Ma, Department of Anesthesiology, First Hospital of Lanzhou University, 1 Dong gang West Road, Lanzhou, Gansu 730000, P.R. China

E-mail: myq2392466@163.com

Key words: giant hamartoma, spontaneous rupture, hemorrhage, pregnancy characteristics and the complications subsequent to its treatment (1). Clinically, RAML is often misdiagnosed as renal cell carcinoma ( $\mathrm{RCC}$ ), and CT examination combined with renal angiography can effectively identify RAML and RCC $(2,3)$. As RAML tends to grow quickly and is often associated with complications including hemorrhage and pain, active intervention is required. RAML is uncommon, with only 22 cases reported over the past 35 years $(4,5)$. RAML complicated with spontaneous rupture and hemorrhage is even more rare in pregnant women. In the present case report, a case of RAML in a 31-year-old pregnant woman with RAML of both kidneys during her second pregnancy was described. The tumor measured $\sim 21 \times 12 \times 10 \mathrm{~cm}$ and grew quickly, causing spontaneous rupture and massive hemorrhage at 20 weeks of gestational age. Emergency exploratory laparotomy, left kidney resection and splenectomy were performed under general anesthesia. A literature review was also performed, and the composition, type classification, imaging characteristics and various treatments currently available for this condition were discussed, in an attempt to provide more information concerning the diagnosis and treatment of this rare renal tumor.

\section{Case report}

A 31-year-old woman at 20 weeks of gestation age was admitted to The First Hospital of Lanzhou University (Lanzhou, China) with the complaint of left flank and abdominal pains for $>3$ days. She was weak, anorexic and severely anemic with episodes of nausea and vomiting. Physical examination revealed tenderness over the left kidney and right lower abdominal regions but with no rebound pain and muscle tension. A giant mass $\sim 10 \mathrm{x} 8 \mathrm{~cm}$ was palpated in the left upper abdomen. Laboratory tests were performed to obtain hemoglobin $(63 \mathrm{~g} / \mathrm{l})$, platelet count $\left(82 \times 10^{9} / 1\right)$, serum urea $(4.07 \mathrm{mmol} / \mathrm{l})$ and serum creatinine $(74.0 \mu \mathrm{mol} / \mathrm{l})$ levels. Computed tomography (CT) plain scan exhibited multiple cystic masses in both kidneys, causing compression on and displacement of the pancreas, spleen and surrounding intestines. The normal morphology and structure of the left kidney had disappeared, and the right kidney was abnormally shaped, with a large mixed density shadow of the soft tissue and fat present (Fig. 1A). Contrast-enhanced CT scan revealed enhancement of multiple vessels supplied by the 


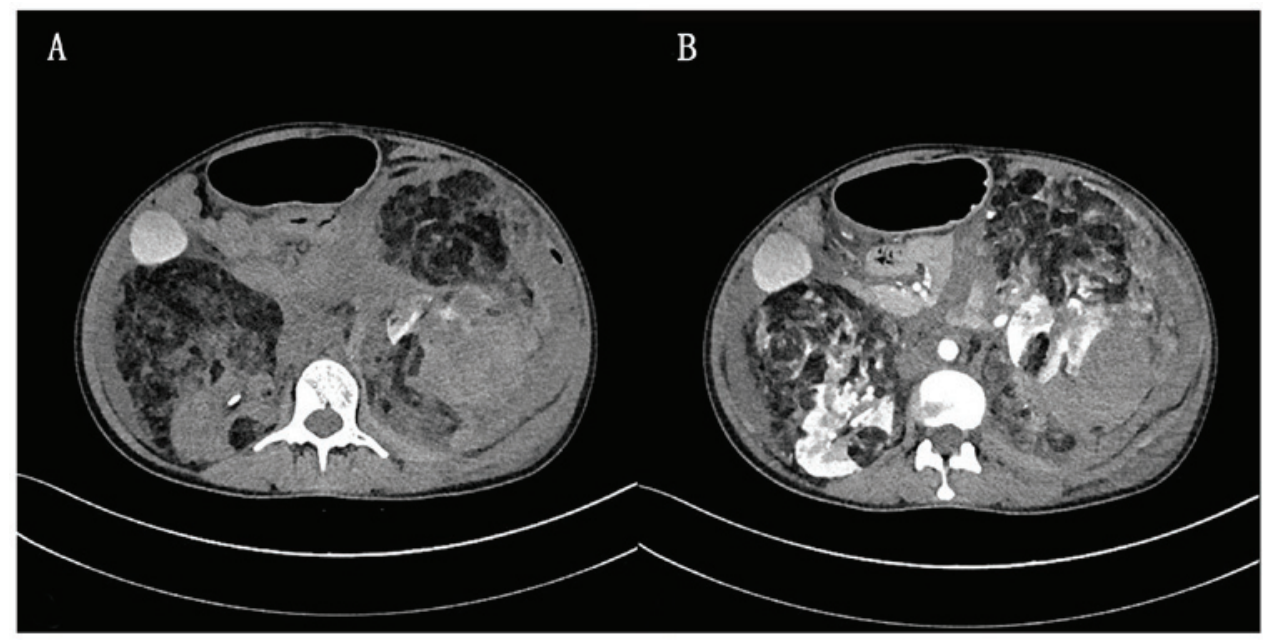

Figure 1. Diagnostic CT scans. (A) CT scans obtained in November 2018 indicated a soft tissue shadow and a fat density shadow in both kidneys, with blood accumulation around the left kidney. (B) Renal parenchyma enhancement during arterial phase with multiple enhanced vascular shadows. CT, computed tomography.

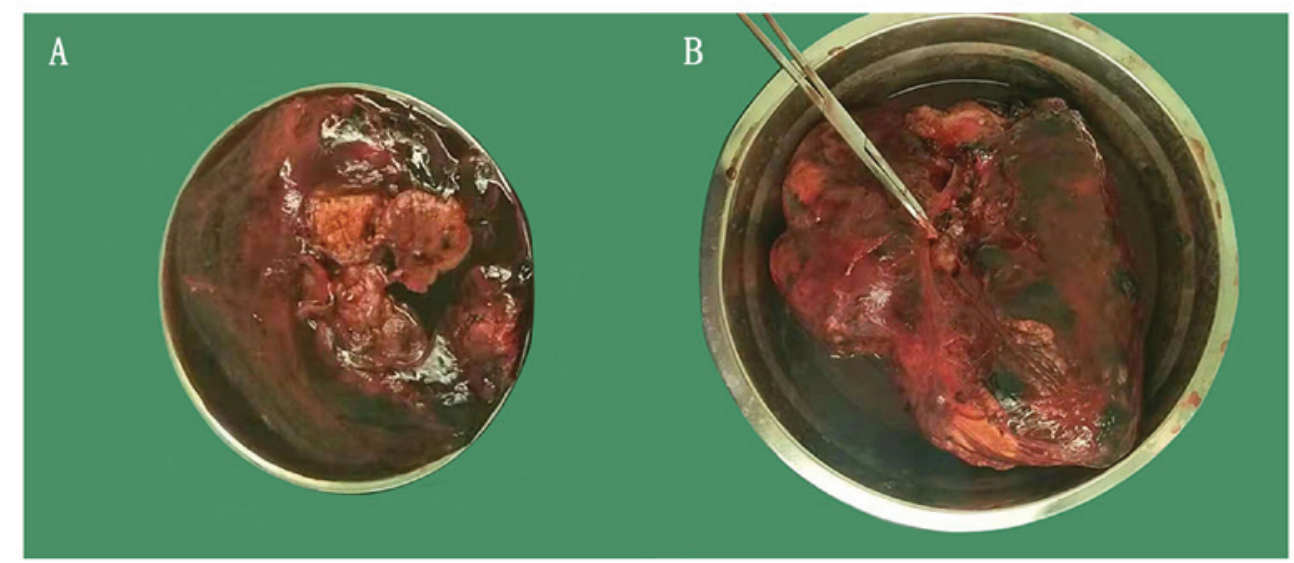

Figure 2. (A) Gross specimen demonstrated that the excised left kidney measured $8 \times 4 \times 4 \mathrm{~cm}$. (B) Gross specimen demonstrated that the excised spleen measured $11 \times 7 \times 4 \mathrm{~cm}$.

bilateral renal artery, fluids in the abdominal and pelvic cavities, destruction of the bilateral renal structures, possible hamartoma, hemorrhage of the left renal lesion, blood accumulation around the left kidney and a small amount of gas accumulation (Fig. 1B). In addition, multiple abnormally enhanced foci were observed in the liver, which were suspected to be hemangioma and multiple cysts; an abnormal enhancement area was observed in the left lobe of the liver, which was considered to be an abnormal perfusion. Magnetic resonance imaging (MRI) was not performed due to the nature of the emergency.

Emergency exploratory laparotomy, left kidney resection and splenectomy were performed under general anesthesia. Postoperative pathology revealed a well-defined grayish yellow and soft mass $\sim 3.0 \times 3.0 \times 2.0 \mathrm{~cm}$ on the surface of the $8.0 \times 4.0 \times 4.0 \mathrm{~cm}$ resection specimen of the left kidney, and a second grayish yellow and soft nodule $\sim 21 \times 12 \times 10 \mathrm{~cm}$ at the renal hilum in the $17 \times 12 \times 9.0 \mathrm{~cm}$ grey-yellow resected specimen (Fig. 2A). A splenectomic specimen measuring $\sim 1 \times 7.0 \times 4.0 \mathrm{~cm}$ was also removed (Fig. 2B). Postoperative pathology of the left kidney revealed that the capsule of the tumor tissue had disappeared due to swelling and infiltration. The tumor parenchyma was composed of a large number of thick-walled blood vessels, and a large amount of fat and a small amount of smooth muscle. The tumor parenchyma cells were not atypical with rare mitosis but hemorrhage and necrosis (Fig. 3A). The splenic capsule and part of the spleen were hemorrhagic, with no tumor tissue observed (Fig. 3B). We performed immunohistochemical analysis of paraffin-embedded sections $(3 \mu \mathrm{m})$, which were fixed with $10 \%$ neutral formaldehyde for $24 \mathrm{~h}$ at room temperature, and subsequently rinsed with tap water. Sections were dewaxed with xylene I and II for $20 \mathrm{~min}$ each, and rehydrated with a descending alcohol series. In addition, samples were incubated with $3 \% \mathrm{H}_{2} \mathrm{O}_{2}$ at room temperature for 5-10 min to block endogenous peroxidase activity and then washed with distilled water three times ( 5 min per wash), followed by heating in a microwave for 10-15 while immersed in PBS. Goat serum was then applied for $15 \mathrm{~min}$ at room temperature. Subsequently, a primary antibody against S-100 was applied (MAB-0697, Fuzhou Maixin Biotechnology Development Co., Ltd.) for $60 \mathrm{~min}$ at room temperature, and washed with PBS. A secondary antibody was then applied (AP, KIT5101; Fuzhou Maixin Biotechnology Development Co., Ltd.) for $15 \mathrm{~min}$ at room temperature. We then performed counterstaining of the sections using hematoxylin for $30 \mathrm{sec}$ at room temperature; the 


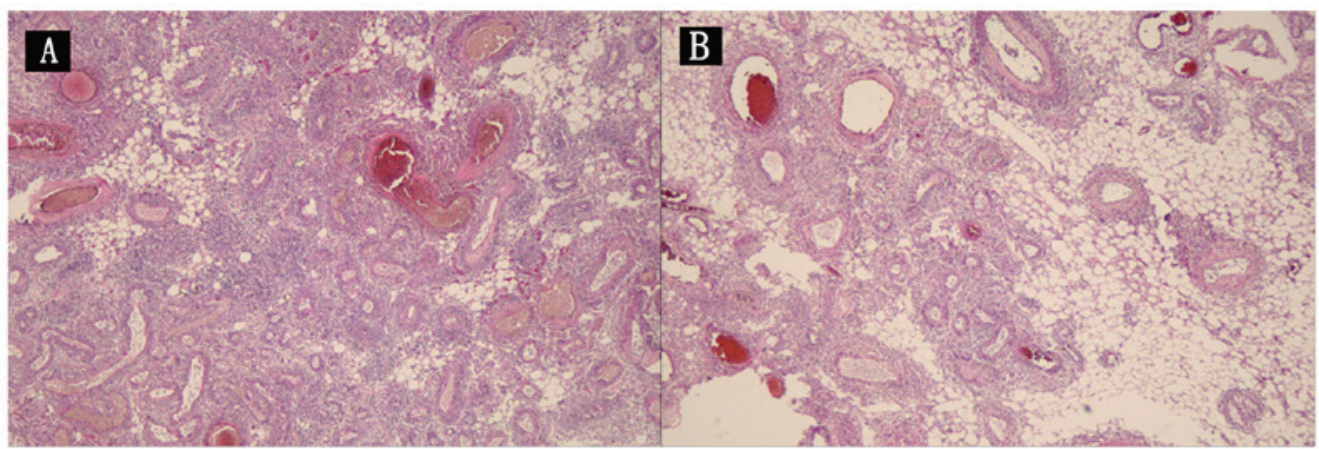

Figure 3. Hematoxylin and eosin staining. (A) Pathological examination demonstrated that the lesion was composed of well-differentiated adipose tissue, smooth muscle and thick-walled vessels. Cells of the tumor parenchyma were not heterogeneous, with rare mitosis (magnification, x200). (B) Pathological examination demonstrated that the splenic capsule and part of the spleen were hemorrhagic, but no tumor tissue observed (magnification, $\mathrm{x} 200$ ).

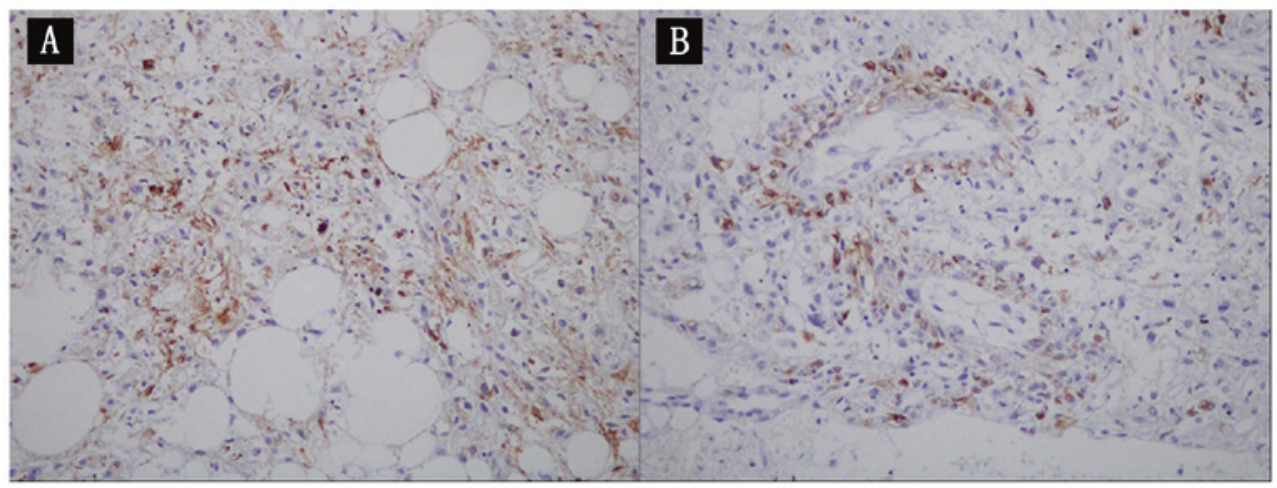

Figure 4. Alkaline phosphatase staining. (A) Immunohistochemical staining demonstrated fine neoplastic smooth muscle, and cells expressing positive staining for the monoclonal antibody HMB-45 associated with melanoma (magnification, x200). (B) Immunohistochemical staining demonstrated that antigen Ki-67 ( $<5 \%$ ), platelet endothelial cell adhesion molecule (vascular+), cluster of differentiation 34 (vascular+), and smooth muscle actin (partial+) (magnification, x200).

sections were sealed with an aqueous mounting agent. Sections were analyzed under a light microscope at x200 magnification. Immunohistochemical staining (alkaline phosphatase method) demonstrated cytokeratin (-), vimentin (1+), S-100 protein (adipocyte+), human melanoma black-45 (scattered lesion+), melanoma antigen recognized by T-cells 1 (1+), antigen Ki-67 $(<5 \%)$, platelet endothelial cell adhesion molecule (vascular+), cluster of differentiation 34 (vascular+), and smooth muscle actin (partial+) staining patterns (Fig. 4A and B). The results of pathology and immunohistochemistry analyses supported the diagnosis of renal hamartoma. Based on these examination results, the patient was diagnosed with giant bilateral renal hamartoma with spontaneous rupture and hemorrhage of the left kidney with involvement of the spleen.

The patient was transferred from the operating room to the Intensive Care Unit for subsequent observation, where she delivered a dead fetus naturally 3 days following surgery, and recovered well postoperatively. After 5 months, the patient was re-admitted and underwent massive tumor resection of the right kidney as part of further treatment, and recovered well postoperatively.

\section{Discussion}

RAML is a benign mesenchymal tumor-like lesion consisting of varying mixtures of smooth muscle, blood vessels and mature fat. It was first described in 1880 (5). It usually occurs in the kidneys but has also been identified in the lung, liver, fallopian tube, vagina and spermatic cord (6). Clinically, RAML may be classified as two types: The tuberous sclerosis complex (TSC) and the sporadic renal hamartoma (7). Patients with TSC-RAML usually have a genetic family history, and the pathogenesis may be associated with mutations in TSC complex subunit 1 and 2 genes. The sporadic renal hamartoma is more common in adults (8). RAML may be diagnosed by ultrasonography, CT and MRI. An overview of the relevant literature may assist in understanding the imaging characteristics of renal hamartoma by different examination methods. For example, renal hamartoma is generally observed as a hyperechoic mass on ultrasound (9). It is difficult to differentiate giant renal hamartoma from other retroperitoneal fatty lesions by ultrasound alone. According to Luo et al (10) and Cheng et al (11) on the imaging characteristics of CT about renal hamartoma, the majority of low fat hamartomas exhibit high density or slightly high density on CT scan, and a few demonstrate slightly low density and isodensity. Cheng et al (11) demonstrated that low-fat renal hamartoma usually exhibited homogeneous low signals on T2 and iso-signal on T1 of MRI plain scan. When the proportion of the fat component is $<20 \%$, it is difficult to demonstrate the imaging features of renal hamartoma. This imaging feature in the clinical diagnosis may assist in the diagnosis of renal hamartoma. However, postoperative pathology remains the gold standard for the diagnosis of renal hamartoma. 
It is clear that early detection and diagnosis of RAML may decrease the occurrence of serious complications. For unruptured hamartoma tumors $>4 \mathrm{~cm}$, minimally invasive procedures including arterial embolization, radiofrequency ablation, cryoablation, mTOR inhibitors, and other novel therapies like partial or total nephrectomy are recommended (12). Pregnancy with renal hamartoma with spontaneous rupture bleeding is rare, and preoperative diagnosis is difficult in pregnant women without presenting clinical symptoms, which often cause misdiagnosis, thereby affecting the safety of the mother and the fetus (13). Pregnancy may promote tumor growth and increase the risk of tumor rupture, which the majority of previous studies hypothesized was due to the ubiquitous expression of estrogen and progesterone receptors in RAML. With the elevation of estrogen and progesterone levels in pregnant women, the tumor tends to grow quickly under stimulation of tumor vascular proliferation, which increases the risk of spontaneous rupture and bleeding of the tumor or surrounding structures $(14,15)$. With the exception of surgical intervention, there is no successful treatment for renal hamartoma complicated with spontaneous rupture and hemorrhage in pregnant women.

To improve the survival rate of patients and fetuses, prenatal examination in the first trimester of pregnancy is important. When the diagnosis is confirmed, interventions including renal artery embolization should be taken positively. At present, for RAML complicated with spontaneous rupture and hemorrhage, surgical resection or angioembolization appears to be the only way to save the life of the patient.

\section{Acknowledgements}

The authors would like to thank Dr Yong-Lin Chen (Department of Pathology, First Hospital of Lanzhou University) for his help in the interpretation of pathological data.

\section{Funding}

The project was supported by the Natural Science Foundation of Gansu Province (grant no. 1606RJZA125).

\section{Availability of data and materials}

All data relevant to the present study are available from the corresponding author on reasonable request.

\section{Authors' contributions}

CMG participated in the collection of the data and the drafting of the manuscript. CY, NX and YM contributed to acquisition and analysis of data, YQM critically revised the manuscript for important intellectual content. All authors have read and approved the final version of this manuscript.

\section{Ethics approval and consent to participate}

Informed consent was obtained from the patient concerning the use of the imaging and other clinical data.

\section{Patient consent for publication}

Informed consent was obtained from the patient concerning the use of the imaging and other clinical data. The patient understood that her name and initials would not be published and due efforts would be made to conceal her identity, though anonymity cannot be guaranteed.

\section{Competing interests}

The authors declare that they have no competing interests

\section{References}

1. Seyam RM, Alkhudair WK, Kattan SA, Alotaibi MF, Alzahrani HM and Altaweel WM: The risks of renal angiomyolipoma: Reviewing the evidence. J Kidney Cancer VHL 4: 13-25, 2017.

2. Catalano OA, Samir AE, Sahani DV and Hahn PF: Pixel distribution analysis: Can it be used to distinguish clear cell carcinomas from angiomyolipomas with minimal fat? Radiology 247 : 738-746, 2008.

3. He ZS, Zhang XC, Zhou LQ, et al: Diagnosis and treatment of renal angiomyolipoma (report of 72 cases). Chin J Urol 23: 135-137, 2002.

4. Preece P, Mees B, Norris B, Christie M, Wagner T and Dundee P: Surgical management of haemorrhaging renal angiomyolipoma in pregnancy. Int J Surg Case Rep 7C: 89-92, 2015.

5. Zhang L, Liu YP and Huang WJ: Analysis of ultrasonic misdiagnosis of atypical renal angiomyolipoma. Shanghai Medical Imaging 18: 288, 2009.

6. Abdulla M, Bui HX, del Rosario AD, Wolf BC and Ross JS: Renal angiomyolipoma. DNA content and immunohistochemical study of classic and multicentric variants. Arch Pathol Lab Med 118: 735-739, 1994.

7. Rijal JP, Dhakal P, Giri S and Dahal KV: Tuberous sclerosis complex with autosomal dominant polycystic kidney disease: a rare duo. BMJ Case Rep 2014: bcr2014207471, 2014

8. Seyam RM, Bissada NK, Kattan SA, Mokhtar AA, Aslam M, Fahmy WE, Mourad WA, Binmahfouz AA, Alzahrani HM and Hanash KA: Changing trends in presentation, diagnosis and management of renal angiomyolipoma: comparison of sporadic and tuberous sclerosis complex-associated forms. Urology 72: 1077-1082, 2008.

9. Wang LL, Dong AJ, Tang JN, Li MB, Yu JJ, Sun PF and Meng GL: Brenge. Diagnosis and treatment of renal hamartoma (40 cases). Mod Oncol 26: 233-237, 2018.

10. Luo J, Jing WB, Zhang DJ, Zhang J and Yang Z: CT and MRI features of renal hamartoma. J Med Imaging (Bellingham) 26: 2043-2046, 2016.

11. Cheng XH, Zhou JZ, Yu ZJ and Zhang JC: Clinical analysis of CT and MRI diagnosis of renal hamartoma and renal cell carcinoma. J Med Imaging 26: 867-869, 2016.

12. Flum AS, Hamoui N, Said MA, Yang XJ, Casalino DD, McGuire BB, Perry KT and Nadler RB: Update on the diagnosis and management of renal angiomyolipoma. J Urol 195: 834-846, 2016.

13. Peng YC and Pan XT: Spontaneous rupture and hemorrhage of pregnancy complicated with renal hamartoma: A case report. Zhejiang Department of Trauma surgery 22: 403-404, 2017.

14. Gould Rothberg BE, Grooms MC and Dharnidharka VR: Rapid growth of a kidney angiomyolipoma after initiation of oral contraceptive therapy. Obstet Gynecol 108: 734-736, 2006.

15. Storm DW and Mowad JJ: Conservative management of a bleeding renal angiomyolipoma in pregnancy. Obstet Gynecol 107: 490-492, 2006.

This work is licensed under a Creative Commons Attribution-NonCommercial-NoDerivatives 4.0 International (CC BY-NC-ND 4.0) License. 